\title{
Lifetime Consumption and Degradation Analysis of the Winding Insulation of Electrical Machines
}

\author{
C. Sciascera*, M. Galea*, P. Giangrande*, C. Gerada* \\ *Faculty of Engineering, University of Nottingham, Nottingham, UK. Email: eexcs8@nottingham.ac.uk
}

Keywords: Thermo-mechanical ageing, multi-stress ageing, winding insulation lifetime, electrical machines.

\begin{abstract}
In this paper, a novel multi-stress model which estimates the lifetime of the winding insulation relative to its duty cycle is proposed and investigated. With an adequate implementation of this model, then an electrical machine can be designed not only in terms of its performance requirements, but also considering the associated reliability and lifetime aspects. Since thermal and thermo-mechanical stresses are considered as the main ageing factors, the model is particularly suited for low voltage, low duty cycle machines. The determination of the model parameters is based on the results of accelerated thermo-mechanical ageing tests, whose procedure is thoroughly reported in the paper. The results of the accelerated ageing tests show that the effect of thermomechanical ageing is significant even for small size, random wound windings under fast temperature rise.
\end{abstract}

\section{Introduction}

It is well known that the More Electric Aircraft (MEA) initiative is resulting in ever-increasing requirements in terms of power density and reliability of the systems involved. An area where this is particularly important, is that of actuation, where electro-mechanical actuators (EMAs) are being increasingly proposed with the aim of substituting the traditional, mechanical and/or hydraulic non-propulsive systems with electrically powered ones, as in [1, 2]. In general, an EMA consists of a compact, high speed, electrical machine with gearing or a ball screw to translate rotational into linear motion [3]. With the increasing power density requirements then one of the main challenges being faced today concerns the ability to maintain the required reliability figures. From an electrical machine perspective this unavoidably involves insulation degradation and lifetime modelling. In [4] a comprehensive review of general approaches used for lifetime modelling of such systems can be found.

Another important aspect of EMA systems is that these are usually operating with low-rate duty cycles (e.g. landing gear extension/retraction and flap actuation), i.e. for short-time or intermittent operations. It is common practice to design the machines for these EMAs with the electro-magnetic limit as the main constraint, while the thermal limitations are considered after hand. This is usually justifiable mainly because, as stated in [5], when operating with a low-rate duty cycle the machine winding will not reach a thermal equilibrium. Considering that stresses will not be constant, any study related to the determination of the lifetime of the insulation will be significantly more complex. As shown in $[3,6]$, in order to ensure the required reliability, it is common practice to design a machine so that the maximum temperature in the slots during the duty cycle is equal to the thermal class temperature of the winding insulation. However, since for EMA applications, the maximum temperature is reached only for a short period, this indicates a potential oversizing of the machine for the specific application. This means that by careful consideration the machine could easily be made to work harder, for example by increasing the current density.

Thus, in this paper, a novel multi-stress model which estimates the lifetime of the motor relative to its particular duty cycle is proposed and investigated, where thermal and thermo-mechanical stresses are considered as the main ageing factors. With this model the machine can be designed not only in terms of its performance (power density) requirements but also taking into account the associated reliability and lifetime aspects. The model is based on the thermal and thermomechanical effects and thus the required parameters are determined from accelerated thermo-mechanical ageing tests, performed on samples of the selected magnetic wire.

While it is common practice to neglect the effect of thermomechanical ageing when small size, random wound wires are considered, however this work shows that this particular aspect can have significant effects when the windings undergo heavy thermal cycles.

\section{The multi-stress ageing model}

In this section, an ageing model which estimates the lifetime of the winding insulation given the stresses applied is presented. Thermal and thermo-mechanical stresses are considered as main ageing factors. Therefore, the model is particularly suited for lifetime estimation of windings under heavy thermal cycling, such as in high performance shorttime duty machines. Indeed, in short-time duty applications the winding temperature typically varies between ambient temperature and a maximum temperature reached at the end of the loading period, without reaching a steady state value. Since the ageing factors considered are only temperature dependent, the only input required by the model is the winding temperature profile $T(t)$, as function of time $t$. 
The lifetime of the insulating material under thermal ageing at constant temperature $T$ can be modelled by (1), where $L_{T}$ is the insulation lifetime under thermal stress at temperature $T$, $L_{0}$ represent the lifetime at a reference temperature $T_{0}$, whilst the parameter $B$ is related to the activation energy of the degradation process.

$$
L_{T}(T)=L_{0} e^{\frac{B}{T}-\frac{B}{T_{0}}}
$$

This model was first introduced in [7] and it relates the thermal ageing to the rate of a temperature dependent chemical reaction, through the use of the Arrhenious equation.

Thermo-mechanical ageing is mainly due to mechanical shear stress between the conductor surface and the insulation. This stress is related to the non-homogeneous temperature distribution in the wire when experiencing thermal cycling, which causes different expansion rates of the conductor and the insulation. The relative thermal expansion between the two materials is proportional both to the difference between their expansion coefficients and to the difference between their temperature [8]. Since the faster the temperature increase is, the less uniform the temperature in the wire will be, then the thermo-mechanical stress can be assumed a function of the change of temperature with respect to time $v$. Due to the mechanical nature of this ageing process, the insulation lifetime under constant thermo-mechanical stress can be modelled with an inverse power law model [8], as shown in (2), where $L_{M}$ is the insulation lifetime of a wire under constant temperature variation $v$, whilst $k$ and $N$ are parameters determined from accelerated ageing tests (described later in the paper).

$$
L_{M}(v)=(1+k|v|)^{-N}
$$

As shown in other works, ageing due to simultaneously applied constant stresses can be accurately modelled by the product of the ageing rate due to the single stresses, with the addition of a proper correction term [4]. Therefore, the lifetime $L$ of a winding under both constant thermal and thermo-mechanical stresses can be represented by (3), where $G$ is the correction term proposed in [4].

$$
\begin{gathered}
L(T, v)=L_{T}(T) L_{M}(v) G(T, v) \\
G(T, v)=(1+k|v|)^{N\left(\frac{B}{T}-\frac{B}{T_{0}}\right)}
\end{gathered}
$$

However, for a given duty cycle, it is not possible to have a winding temperature profile whose temperature and temperature variation are both constant, except when the temperature variation is null. Therefore, in order to propose a model which relates the winding lifetime to its thermal cycle, it is necessary to find a local form of the model which estimates the instantaneous life consumption at time $t$, given the temperature $T(t)$ and its time derivative $v(t)$.
In order to achieve this, a function $f(t)$ is defined, which represents the insulation life remaining at time $t$ if its temperature is kept constant at $T_{0}$. This function is $f\left(t_{0}\right)=L_{0}$ at the initial time $t_{0}$ where there has not been any ageing and $f\left(t_{f}\right)=0$ at the time of failure $t_{f}$, where the whole insulation lifetime has been consumed. The function $f(t)$ will thus decrease with respect to time according to (5).

$$
\frac{d f}{d t}(t)=-\frac{L_{0}}{L(T(t), v(t))}
$$

By integrating (5), a relationship between the winding lifetime $t_{f}$ and its temperature profile is finally obtained, and this is described by (6).

$$
\int_{t_{0}}^{t_{f}} \frac{1}{L(T(t), v(t))} d t=1
$$

\section{Model simulation for short-time duty}

Using (6), it is possible to estimate the lifetime of the insulation given the temperature profile of the winding. In particular, in the case of short-time duty applications, given the temperature profile during the duty cycle, the life consumed over each cycle will be constant. The number of cycles $C$ that the insulation will be able to withstand can be determined from (7), where $t_{1}$ and $t_{2}$ are the initial and final duty cycle times, respectively. Therefore, the insulation lifetime is obtained by multiplying $C$ for the duty cycle's time length.

$$
C=\frac{1}{\int_{t_{1}}^{t_{2}} \frac{1}{L(T(t), v(t))} d t}
$$

Some preliminary investigations of the proposed model are done, with the objective of understanding the effects that the key parameters have on the predicted lifetime. For these tests, the duty cycle shown in Figure 1 is considered, where the temperature varies between temperature $T_{1}$ and $T_{2}$, with a profile similar to the operating one.

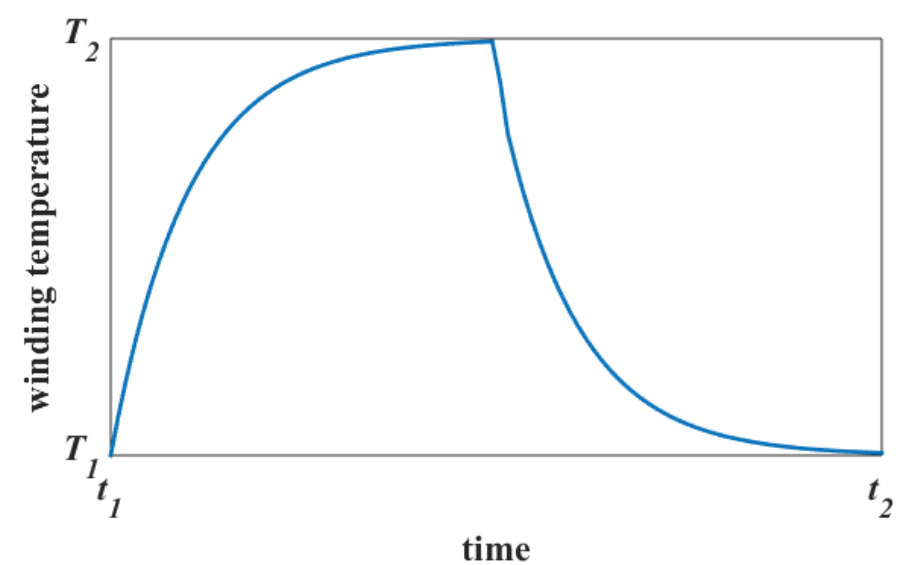

Figure 1: Temperature profile during the motor duty cycle. 
Figure 2 shows how the estimated winding lifetime varies when the minimum temperature $T_{1}$ and the maximum temperature $T_{2}$ are fixed and only the duty cycle interval $t_{2}-t_{1}$ is changed. In particular, different values of the parameter $k$ of (2) are considered. As expected, for $k=0$ (i.e. the case where thermo-mechanical ageing is neglected), the calculated lifetime is not affected by the duty cycle time and remains constant. On the other hand, as $k$ is increased, the lifetime will start to be reduced. For faster duty cycles the insulation lifetime will be shorter because of the faster temperature variation.

Similar results are obtained by varying the value of parameter $N$ in (2), as shown in Figure 3, where $N=0$ is the case when thermo-mechanical ageing is neglected, whilst as $N$ increases, the effects of thermo-mechanical ageing become more significant.

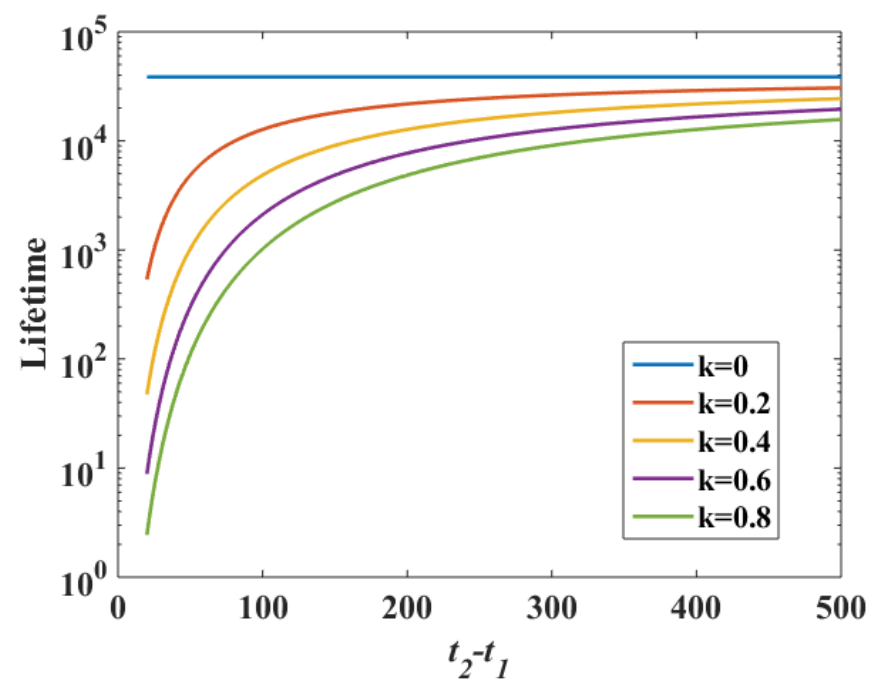

Figure 2: Lifetime vs duty cycle period, for different values of $k$, for fixed temperatures $T_{1}$ and $T_{2}$.

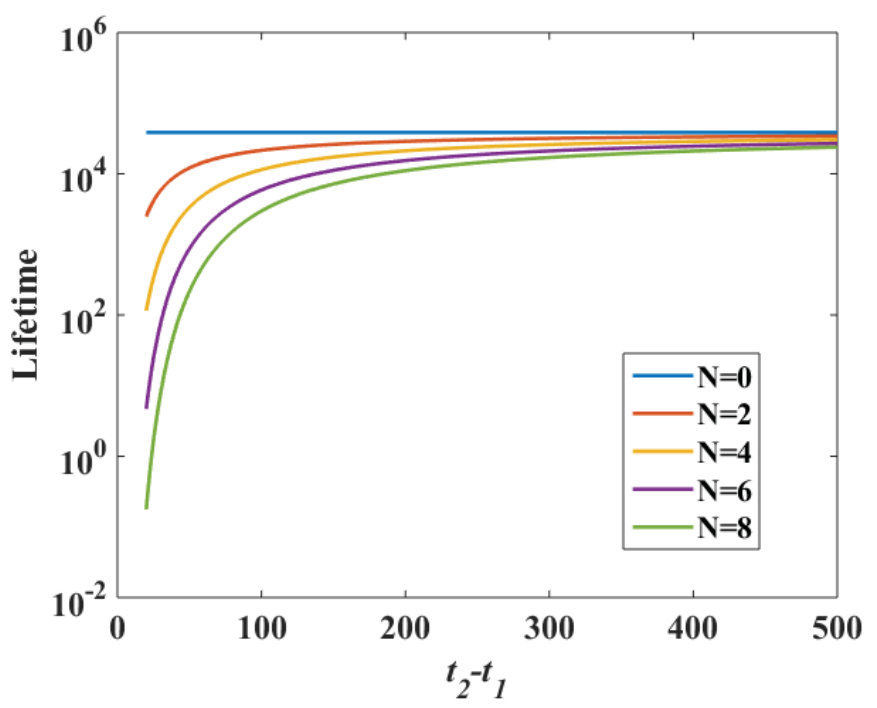

Figure 3: Lifetime vs duty cycle period, for different values of $N$, for fixed temperatures $T_{1}$ and $T_{2}$.
As can be perceived from all the above, a critical aspect for the correct operation of the model is the correct definition and tuning of the free parameters $k$ and $N$. These parameters are derived from the results of specific accelerated ageing tests, where appropriate tuning methods are applied. This is described in Section 4.

\section{Accelerated thermo-mechanical ageing test}

In this section, the procedure of the accelerated thermomechanical ageing tests conducted for the determination of the ageing model parameters is reported. In particular, the scope of the experiment is the estimation of the parameters $k$ and $N$ of (2). These parameters are crucial for the model accuracy. Indeed, while the parameters of the thermal ageing model in (1) can be derived from the expected lifetime vs temperature curve usually provided by the wire manufacturer and which are based on accelerated thermal ageing tests, usually there is no data available for what concerns the thermo-mechanical ageing.

In the experiment, a class 200 , conductor diameter $0.56 \mathrm{~mm}$, round enamelled copper wire was tested. The base coat material of the wire insulation is modified polyesterimide. For each test, six identical specimens are aged, as suggested in [9], in order to get statistically significant results. Each specimen is composed of two identical coils wound around the same tooth of the stator of the electric motor for the EMA described in [1]. The coils are subjected to thermal cycles limited between two specific temperatures.

In order to approximate the real operating conditions as much as possible, the required thermal conditions are achieved by exploiting the temperature rise due to the inherent ohmic losses of the copper rather than using an oven, as suggested in [10].

Figure 4 shows the test set-up which allows for the required thermal cycles to be performed by applying a DC voltage to the specimens connected in series with a programmable DC power supply.

The specimens are evenly distributed in the stator to ensure symmetry and thus achieve similarity between them all. Figure 5 illustrates how the specimens are located in the machine stator.

The temperature in the coils was measured using K-type thermocouples. One sensor for each specimen was used, with it being placed in the predicted location of the maximum hot spot temperature and used to achieve active DC voltage control, relative to the desired temperature profile.

In order to determine the lifetime of the specimens it is necessary to select a pass/fail criteria. The insulation resistance of a specimen is a good indicator of deterioration. Therefore, the resistance between two coils of the same specimen undergoing thermal cycles was periodically measured with a mega-ohmmeter. The resistance of the specimens was noticed to be typically higher than $1 \mathrm{~T} \Omega$ for most of the specimen's life, with a significant resistance drop near the end of the specimen's lifetime (i.e. when its insulation is not able to withstand the rated voltage). Considering this, then it was decided to set the end of a specimen's life when its insulation resistance is below $1 \mathrm{G} \Omega$. 


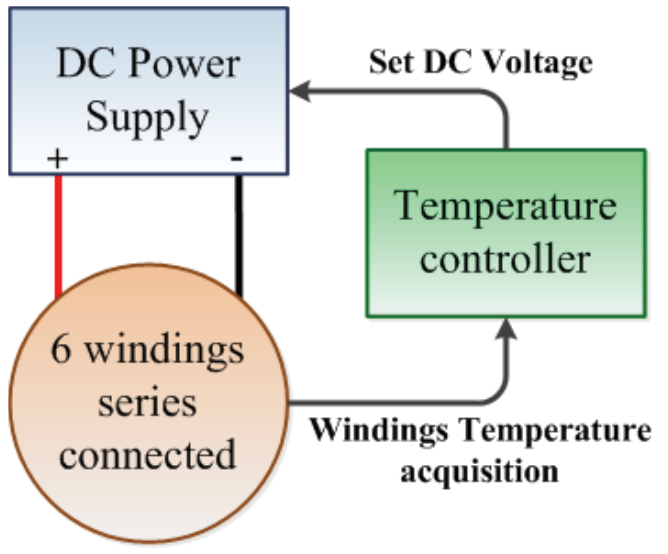

Figure 4: Accelerated thermo-mechanical ageing test scheme.

As explained in [11], the time to failure of the tested wire under thermal and thermo-mechanical stresses for a specific duty cycle can be represented by the mean of the time to failure $\mu$ of the single specimens. Supposing that the data collected is normally distributed, it is then possible to calculate the approximated variance in order to get a confidence interval of the results obtained.

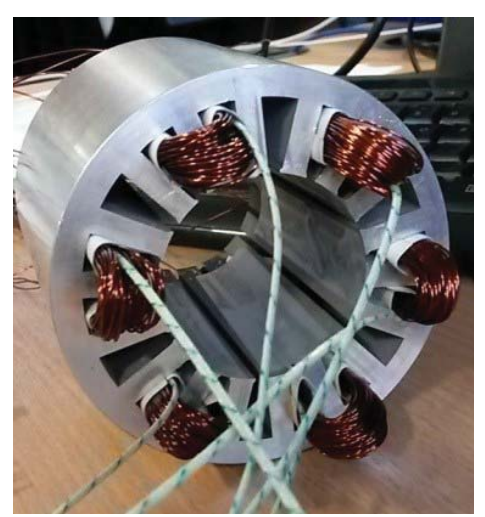

Figure 5: Specimens placed in the stator for the ageing tests.

\section{Experimental results and model's parameters estimation}

An accelerated thermo-mechanical ageing test was conducted using the experimental setup introduced in the previous section. The specimens were cycled between $210^{\circ} \mathrm{C}$ and $285^{\circ} \mathrm{C}$. Each thermal cycle was 28 minutes long. Figure 6 shows the temperature profile recorded during a single cycle. As reported In Table 1, the average time to failure observed from the accelerated ageing test is 90 hours. The insulation resistance, whose value was used as pass/fail criteria, was measured every 24 hours. The $95 \%$ confidence interval of the results is between 75.9 and 104.1 hours.

The experimental results show the importance of considering thermo-mechanical ageing along with thermal ageing in the insulation lifetime estimation, even for small size, random wound wires, when the temperature variation is fast. Indeed, if in the lifetime model only thermal ageing was taken into account (i.e. when the parameter $k$ is equal to 0 in (2)), then the failure of the specimens would have been expected after 713 hours for the same thermal cycle.

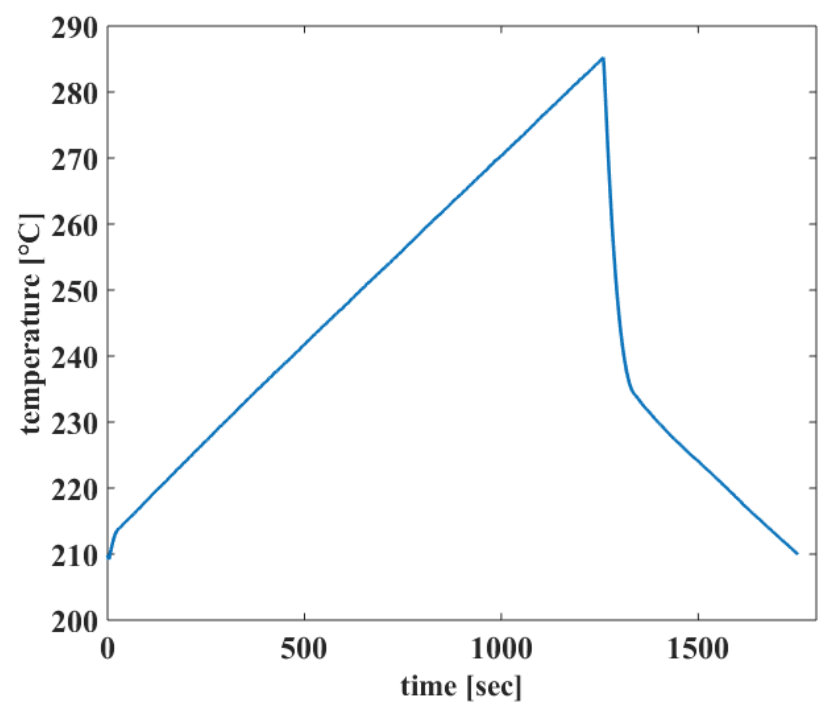

Figure 6: Temperature profile measured during a thermal cycle.

\begin{tabular}{|c|c|}
\hline Experimental average lifetime & 90 hours \\
\hline $\mathbf{9 5 \%}$ confidence interval of the results & $75.9 / 104.1$ hours \\
\hline $\begin{array}{c}\text { Expected lifetime neglecting thermo- } \\
\text { mechanical ageing }\end{array}$ & 713 hours \\
\hline
\end{tabular}

Table 1: Average lifetime collected from the accelerated ageing test. Confidence interval of the results. Expected lifetime if only thermal ageing was considered.

\subsection{Fine tuning the model's parameters}

The results of the first accelerated ageing test performed can be used for a first tuning of the free parameters of the lifetime model presented in (6). This can be done by considering all the possible values of the free parameters $k$ and $N$ that, when used in the lifetime model, would ensure a match with the experimental results from the accelerated ageing test.

Figure 7 shows the values of $k$ and $N$ which results in a lifetime prediction (for the specific thermal cycle) of 90 hours, thus matching the experimentally obtained value.

As the lifetime model comprises two free parameters, then in order to achieve complete characterization of the proposed lifetime model, an accelerated ageing test with a different thermal cycle would need to be performed. From this additional test another curve as the one of Figure 7 can be calculated and its intersection with the curve shown in Figure 7 would provide a unique combination of the free parameters $k$ and $N$ that can then be used for any duty cycle. 


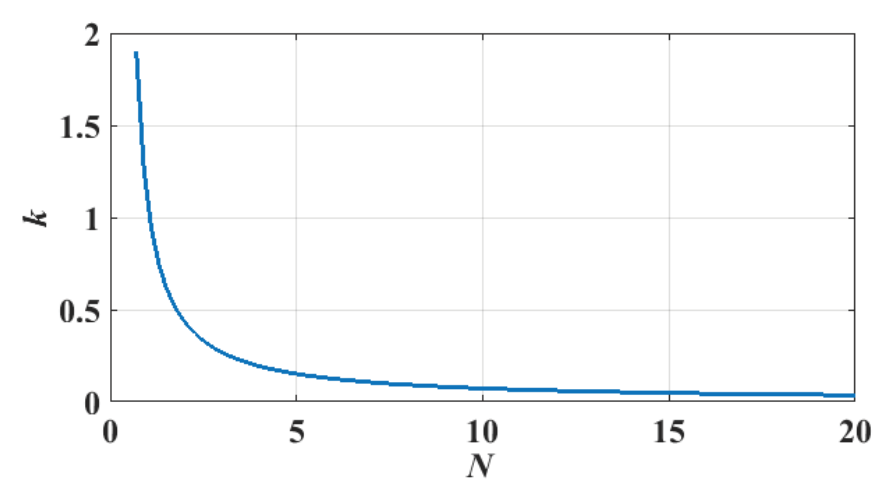

Figure 7: Values of coefficients $k$ and $N$ for which model lifetime expectation and experimental results coincide.

\section{Conclusions}

A novel multi-stress model for the estimation of electrical machines' insulation lifetime under thermal and thermomechanical stresses has been proposed. This model is particularly suited for low voltage, low duty cycle, high performance motors. An appropriate implementation of the model can significantly improve the motor design especially when considering the reliability aspects in parallel to performance maximization.

A procedure for tuning the model parameters has been proposed, which derives from the experimental results of accelerated ageing tests.

The ageing tests presented in this paper show that when heavy thermal cycles are necessary, then the ageing effects due to thermo-mechanical aspects need to be included, even for small size, random wound specimens.

Further ageing tests will allow for a complete tuning of the model parameters and the model will then be used for improving the design of a motor for a particular electromechanical actuator.

On the base of the first experiment performed, some considerations can be done in order to improve the procedure of future tests.

Even if, as suggested in [9], five specimens would be sufficient for achieving statistically significant results, the practical difficulty of reproducing identical specimens (random wound windings) indicates that increasing the number of specimens would improve the accuracy of the results. In this way, the confidence interval of the results would be narrowed.

A perceived difficulty in the above work is related to the use of a complete machine stator, which led to irregular temperature distribution. Thus in future experiments, the specimens will be mounted on identical motorettes similar to the ones proposed in [12], which means that the specimens will be thermally insulated from each other.

Since it is always very difficult to correctly predict the exact location of the windings hot spot then in the next tests, one of the specimens will be equipped with an array of evenly distributed thermocouples. This will improve the accuracy of the winding hot spot detection.

Finally, in order to improve the pass/fail criteria, the measurement of the insulation dissipation factor and capacitance will be added to the resistance measurement in order to enhance the accuracy in the detection of degradation of the insulation physical properties.

\section{References}

[1] C. Sciascera, P. Giangrande, C. Brunson, M. Galea, and C. Gerada, "Optimal Design of an Electro-Mechanical Actuator for Aerospace Application," in Industrial Electronics Society, 2015. 41st Annual Conference of IEEE, 2015, p. 6 pp.

[2] C. Gerada and K. J. Bradley, "Integrated PM Machine Design for an Aircraft EMA," Industrial Electronics, IEEE Transactions on, vol. 55, pp. 3300-3306, 2008.

[3] M. Galea, G. Buticchi, L. Empringham, L. de Lillo, and C. Gerada, "Design of a High-Force-Density Tubular Motor," Industry Applications, IEEE Transactions on, vol. 50, pp. 2523-2532, 2014.

[4] G. C. Montanari and L. Simoni, "Aging phenomenology and modeling," Electrical Insulation, IEEE Transactions on, vol. 28, pp. 755-776, 1993.

[5] IEC, "Rotating Electrical Machines Part I: Rating and Performance," in 60034-1, ed, 2010.

[6] M. Galea, C. Gerada, T. Raminosoa, and P. Wheeler, "A Thermal Improvement Technique for the Phase Windings of Electrical Machines," Industry Applications, IEEE Transactions on, vol. 48, pp. 79-87, 2012.

[7] T. W. Dakin, "Electrical Insulation Deterioration Treated as a Chemical Rate Phenomenon," American Institute of Electrical Engineers, Transactions of the, vol. 67, pp. 113-122, 1948.

[8] V. I. J. Kokko, "Ageing due to thermal cycling by start and stop cycles in lifetime estimation of hydroelectric generator stator windings," in Electric Machines \& Drives Conference (IEMDC), 2011 IEEE International, 2011, pp. 318-323.

[9] IEC, "Rotating Electrical Machines Part 18-31: Thermal Evaluation and Classification of Insulation Systems Used in Rotating Machines," ed. 60034-18-31, 2012.

[10] "IEEE Recommended Practice for Thermal Cycle Testing of Form-Wound Stator Bars and Coils for Large Rotating Machines" IEEE Std 1310-2012 (Revision of IEEE Std 1310-1996, pp. 1-58, 2012.

[11] IEC, "Guide for the statistical analysis of ageing test data Part 1: Methods based on mean values of normally distributed test results," ed. 60493-1, 2011.

[12] IEC, "Rotating electrical machines Part 18-21: Functional evaluation of insulation systems- Test procedures for wire-wound windings - Thermal evaluation and classification.," in 60034-18-21, ed, 2013. 